\title{
Theoretical framework for classification and prediction of ultrafast and strong-field phenomena in solids
}

\author{
Stanislav Yu. Kruchinin ${ }^{1, *}$, Ferenc Krausz ${ }^{2}$, and Vladislav S. Yakovlev ${ }^{1}$ \\ ${ }^{1}$ University of Vienna, Faculty of Physics and Center for Computational Materials Sciences, \\ Sensengasse 8/12, 1090 Vienna, Austria \\ ${ }^{2}$ Max Planck Institute of Quantum Optics, Hans-Kopfermann-Str. 1, 85748 Garching, Germany \\ ${ }^{3}$ Ludwig Maximilian University of Munich, Am Coulombwall 1, 85748 Garching, Germany
}

\begin{abstract}
We study the characteristic energy and time scales describing the coherent electron dynamics and decoherence phenomena in solids interacting with ultrashort laser pulses. Our analysis resulted in the derivation system of dimensionless adiabaticity parameters and derivation of the non-Markovian density-matrix equations applicable on arbitrary short timescales.
\end{abstract}

In our recent work [1], we systematically applied theoretical methods of adiabatic perturbation theory $[2,3]$ to study the problem of electron in the periodic potential driven by an external electric field. Interaction regimes of this system can be completely classified with a set of dimensionless adiabaticity parameters (see Tables 1 and 2), each of which is a ratio of two characteristic frequencies.

Table 1. Characteristic frequencies and dimensionless adiabaticity parameters describing the regimes (adiabatic and diabatic limits) of laser-field interaction with periodic potentials. The parameters are proportional to the ratio of the frequencies from upper row and left column.

\begin{tabular}{c|cccc}
\hline \hline & $E_{g}$ & $U_{\mathrm{p}}$ & $\omega_{\mathrm{B}}$ & $\omega_{\mathrm{R}}$ \\
\hline$\omega_{0}$ & $N$ & $\gamma_{\mathrm{NP}}$ & $\gamma_{\mathrm{DL}}$ & $\gamma_{\mathrm{RF}}^{(0)}$ \\
$E_{\mathrm{g}}$ & & $\gamma_{\mathrm{K}}^{-2}$ & $\gamma_{\mathrm{BH}}$ & $\gamma_{\mathrm{RF}}^{(\mathrm{g})}$ \\
$U_{\mathrm{p}}$ & & & $\gamma_{\mathrm{BP}}$ & $\gamma_{\mathrm{RP}}$ \\
$\omega_{\mathrm{B}}$ & & & & $\gamma_{\mathrm{RB}}$ \\
\hline \hline
\end{tabular}

Table 2. Acronyms of adiabaticity parameters and their meanings.

\begin{tabular}{l|l}
\hline \hline NP & NonPerturbative intensity [4] \\
DL & Dynamic Localization [5] \\
K & Keldysh [6] \\
RF & Rabi Flopping [7] \\
BH & Band Hybridization [8] \\
BP & Bloch-to-Ponderomotive \\
RP & Rabi-to-Ponderomotive \\
RB & Rabi-to-Bloch \\
\hline \hline
\end{tabular}

Here, $N$ is the order of the lowest possible multiphoton transition, $E_{\mathrm{g}}$ is the minimal direct bandgap, $U_{\mathrm{p}}=U_{\mathrm{p}}^{(\mathrm{c})}(0)-U_{\mathrm{p}}^{(\mathrm{v})}(0), U_{\mathrm{p}}^{(n)}(\mathbf{k})=\overline{E_{n}(\mathbf{K}(t))}-E_{n}(\mathbf{k})$ is the ponderomotive energy

*Corresponding author: stanislav.kruchinin@univie.ac.at 
of electron in the band $n$ with dispersion $E_{n}(\mathbf{k}), \overline{E_{n}(\mathbf{K}(t))}$ is the cycle-averaged energy of electron, $\mathbf{K}(t)=\mathbf{k}+\mathbf{A}(t)$ is the kinetic crystal momentum, $\mathbf{A}(t)=-\int_{t_{0}}^{t} d t_{1} \mathbf{F}\left(t_{1}\right)$ is the vector potential of the laser field $\mathbf{F}(t), \omega_{\mathrm{B}}=\left|F_{0} a\right|$ is the peak Bloch frequency, $a$ is the lattice constant, $\omega_{\mathrm{R}}=\left|\mathbf{F}_{0} \cdot \xi_{\mathrm{cv}}^{(\max )}\right|$ is the peak Rabi frequency, $\left|\xi_{\mathrm{cv}}^{(\max )}\right|=\max _{\mathbf{k}}\left|\xi_{\mathrm{cv}}(\mathbf{k})\right|$ is the peak absolute value of the interband matrix element.

Each parameter defines the adiabatic and diabatic limits, where one of the characteristic frequencies is dominant, and thus allows for effective time scale separation by using the adiabatic (field-dressed) or diabatic (field-free) basis.

Time-dependent perturbative Dyson series constructed in these two bases are dual, i.e. their small parameters are inversely proportional to each other. In particular, the Keldysh parameter $\gamma_{\mathrm{K}}$ emerging from the perturbation theory constructed in the Houston basis is inversely proportional to the Rabi flopping parameter $\gamma_{\mathrm{RF}}^{(0)}$, which appears in the theory employing the Bloch basis

$$
\gamma_{\mathrm{K}}=\frac{1}{2 \gamma_{\mathrm{RF}}^{(0)}} .
$$

All real-world quantum systems are connected to some dissipative environment and experience an irreversible decay of quantum coherence and state population. Thus, analysis of characteristic timescales for relaxation processes is essential for both fundamental and applied research. Recent numerical simulations of high-harmonic generation in solids [10, 11] have shown that unphysically fast pure dephasing times $T_{2} \sim 1-3 \mathrm{fs}$ are required to reach an agreement with experimental data, if the modelling is performed in the independent-particle and Markov approximations. Our estimations of correlation decay time $\tau_{\mathrm{B}}$ for electron-electron and electron-phonon interactions in semiconductors at room temperature have shown that it is comparable to typical pulse durations (from units to tens of femtoseconds). This implies that scattering processes cannot be considered as instantaneous events. The characteristic time scales and applicability conditions of common approximations for dielectrics exposed to a few-cycle visible/near-infrared pulse (VIS/NIR) are summarized in the Tables 3 and 4, respectively [12].

Table 3. Characteristic time scales of the laser-matter interaction problem in the Houston basis and their typical values for the bath of phonons (ph) and carrier correlations (c). The parameters are estimated for the $\alpha$-quartz ( $E_{\mathrm{g}}=9 \mathrm{eV}, E_{\mathrm{ex}}=1.2 \mathrm{eV}, E_{\mathrm{LO}}=0.153 \mathrm{eV}$ ) and VIS/NIR laser pulses

in the wavelength range $400-2500 \mathrm{~nm}$.

\begin{tabular}{lll}
\hline \hline Time scale & Denotation & Values \\
\hline Optical cycle & $T_{0}=2 \pi / \omega_{0}$ & $1.4-8.4 \mathrm{fs}$ \\
Pulse duration & $\tau_{\mathrm{L}}$ & $0.7-40 \mathrm{fs}$ \\
Elapsed time & $t_{\max }$ & $\sim \tau_{\mathrm{L}}$ \\
Minimal bandgap & $\tau_{\mathrm{g}}=2 \pi /\left(E_{\mathrm{g}}+U_{\mathrm{p}}\right)$ & $0.23-0.46 \mathrm{fs}$ \\
Change of adiabatic energies & $\tau_{\mathrm{AE}}$ & $\gtrsim 0.1 \mathrm{fs}$ \\
Change of adiabatic states & $\tau_{\mathrm{AS}}$ & $1-8 \mathrm{fs}$ \\
Minimal relaxation time & $\tau_{\mathrm{R}}$ & $\gtrsim 27 \mathrm{fs}(\mathrm{ph}), \sim 4 \mathrm{fs}(\mathrm{c})$ \\
Bath correlation decay time & $\tau_{\mathrm{B}}$ & $\gtrsim 10 \mathrm{fs}(\mathrm{ph}), \sim 2 \mathrm{fs}(\mathrm{c})$ \\
\hline \hline
\end{tabular}


Table 4. Summary of approximations applicability for the bath of carrier correlations.

\begin{tabular}{lll}
\hline \hline Approximation & Conditions & Applicability \\
\hline Weak coupling (Born) & $\tau_{\mathrm{B}} \ll \tau_{\mathrm{R}}$ & Yes \\
Secular & $\tau_{\mathrm{g}} \ll \tau_{\mathrm{R}} ; \tau_{\mathrm{g}} \ll \tau_{\mathrm{AE}}, \tau_{\mathrm{AS}}$ & Partial \\
Instantaneous eigenbasis & $\tau_{\mathrm{B}} \ll \tau_{\mathrm{AE}}, \tau_{\mathrm{AS}}$ & Partial \\
Markov & $\tau_{\mathrm{B}} \ll t_{\max }$ & No \\
\hline \hline
\end{tabular}

The theory open quantum systems [13] suggests the master equations with effective time-dependent relaxation rates taking into account finite correlation decay time and memory effects. In this approach, the equations of motion are generalized into the nonMarkovian regime by using the time-dependent decoherence rates $\gamma_{n m, \mathbf{k}}(t)$ [12]:

$$
\begin{aligned}
\frac{d}{d t} \rho_{n m, \mathbf{k}}(t) & =-\gamma_{n m, \mathbf{k}}(t) \rho_{n m, \mathbf{k}}(t)+i \Omega_{n m, \mathbf{k}}^{*}(t)\left[\rho_{n n, \mathbf{k}}(t)-\rho_{m m, \mathbf{k}}(t)\right] \\
& -i \sum_{l \neq n, m}\left[\Omega_{n l, \mathbf{k}}^{*}(t) \rho_{l m, \mathbf{k}}-\Omega_{l m, \mathbf{k}}(t) \rho_{n l, \mathbf{k}}(t)\right], \\
\frac{d}{d t} \rho_{n n, \mathbf{k}}(t)= & i \sum_{l \neq n} \Omega_{n l, \mathbf{k}}(t) \rho_{n l, \mathbf{k}}(t)+\text { c. c. }
\end{aligned}
$$

where $\Omega_{n m, \mathbf{k}}(t)=\mathbf{F}(t) \cdot \boldsymbol{\xi}_{n m}(\mathbf{K}(t)) \exp \left[i \phi_{n m}^{\prime}(\mathbf{k}, t)\right]$ is the matrix element of interband interaction,

$$
\phi_{n m}^{\prime}(\mathbf{k}, t)=\int_{t_{0}}^{t} E_{n m}^{\prime}\left(\mathbf{K}\left(t_{1}\right)\right) \mathrm{d} t_{1},
$$

is the change of a total phase between the Houston states of the bands $n$ and $m$, $E_{n m}^{\prime}(\mathbf{K}(t))=E_{n}^{\prime}(\mathbf{K}(t))-E_{m}^{\prime}(\mathbf{K}(t)), E_{n}^{\prime}(\mathbf{K}(t))=E_{n}(\mathbf{K}(t))+\mathbf{F}(t) \cdot \boldsymbol{\xi}_{n n}(\mathbf{K}(t))$, and $\boldsymbol{\xi}_{n n}(\mathbf{K}(t))$ is the Berry connection of the $n$th band.

This work was supported by the DFG Cluster of Excellence: Munich-Centre for Advanced Photonics (MAP). S. Yu. K. acknowledges support from the Austrian Science Fund (FWF) within the Lise Meitner Project No. M2198-N30.

\section{References}

1. S. Yu. Kruchinin, F. Krausz, and V. S. Yakovlev, Rev. Mod. Phys. 90, 021002 (2018).

2. A. Bohm, A. Mostafazadeh, H. Koizumi, Q. Niu, and J. Zwanziger, The Geometric Phase in Quantum Systems: Foundations, Mathematical Concepts, and Applications in Molecular and Condensed Matter Physics (Springer Berlin Heidelberg, 2013).

3. A. Mostafazadeh, Phys. Rev. A. 55, 1653-1664 (1997).

4. H. R. Reiss, Prog. Quant. Electron. 16, 1-71 (1992).

5. D. H. Dunlap and V. M. Kenkre, Phys. Rev. B 34, 3625-3633 (1986).

6. S. Hughes, Phys. Rev. Lett. 81, 3363-3366 (1998).

7. L. V. Keldysh, Sov. Phys. JETP 20, 1307 (1965).

8. G. Bastard, R. Ferreira, S. Chelles, and P. Voisin, Phys. Rev. B 50, 4445-4449 (1994).

9. M. Frasca, Phys. Rev. A 58, 3439-3442 (1998).

10. G. Vampa, T. J. Hammond, N. Thire, B. E. Schmidt, F. Legare, C. R. McDonald, T. Brabec, D. D. Klug, and P. B. Corkum, Phys. Rev. Lett. 115, 193603 (2015).

11. T. T. Luu, M. Garg, S. Yu. Kruchinin, A. Moulet, M. Th. Hassan, and E. Goulielmakis, Nature 521, 498-502 (2015).

12. S. Yu. Kruchinin, arXiv:1806.05556 (2018).

13. H.-P. Breuer and F. Petruccione, The Theory of Open Quantum Systems (Oxford University Press, 2002). 